\title{
First-line pembrolizumab monotherapy for metastatic PD-L1-positive NSCLC: real-world analysis of time on treatment
}

\author{
Vamsidhar Velcheti*,1, Sheenu Chandwani ${ }^{2}$, Xin Chen², M Catherine Pietanza² \& Thomas \\ Burke ${ }^{2}$ \\ ${ }^{1}$ NYU Langone, Perlmutter Cancer Center, 160 E 34th St, New York, NY 10016, USA \\ ${ }^{2}$ Merck \& Co., Inc., 2000 Galloping Hill Rd., Kenilworth, NJ 07033, USA \\ *Author for correspondence: Tel.: +1 212731 6000; Fax.: +1 212731 5591; vamsidhar.velcheti@nyulangone.org
}

\begin{abstract}
Aim: To determine real-world time on treatment (rwToT) with first-line pembrolizumab monotherapy for metastatic non-small-cell lung cancer (NSCLC) with programmed death ligand-1 (PD-L1) tumor proportion score (TPS) $\geq 50 \%$. Methods: The Kaplan-Meier rwToT was estimated from electronic health record data for adults who initiated first-line pembrolizumab monotherapy for stage IV, PD-L1 TPS $\geq 50 \%$ NSCLC, with negative/unknown EGFR/ALK aberrations, and $\geq 6$ months' follow-up until database cutoff. Results: A total of 386 patients with ECOG $0-1$ had a median rwToT of 6.9 months ( $95 \% \mathrm{Cl}: 5.6-8.3$ ) and 12-month on-treatment rate of $36.4 \%$ (31.2-41.6) versus $40.3 \%$ (32.5-47.9) and $37.6 \%$ (31.9-43.4) in KEYNOTE-024 (KN024) and KN042 (stage IV/TPS $\geq 50 \%$ subpopulation), respectively. The 24-month restricted-mean rwTOT (extrapolated) was 10.5 months (9.4-11.7), versus 11.0 (9.5-12.5) and 10.4 (9.3-11.5) in KN024 and KN042, respectively. Conclusion: First-line pembrolizumab monotherapy rwToT in metastatic PD-L1 TPS $\geq 50 \%$ NSCLC for trial-matched patients is similar to treatment duration in KNO24 and KN042.
\end{abstract}

First draft submitted: 15 April 2019; Accepted for publication: 28 May 2019; Published online: 11 June 2019

Keywords: database • first-line monotherapy • metastatic non-small-cell lung cancer • PD-L1 tumor proportion score $\bullet$ pembrolizumab • real world $\bullet$ time on treatment

Pembrolizumab monotherapy was approved in the USA in October 2016 for first-line treatment of metastatic nonsmall-cell lung cancer (NSCLC) with high expression of programmed death ligand-1 (PD-L1 tumor proportion score [TPS] $\geq 50 \%$ ) based on the findings of the KEYNOTE-024 (KN024) clinical trial [1]. The currently labeled pembrolizumab dosage for treatment of NSCLC, as of April 2019 [2] and applicable to all approved NSCLC indications, is ' $200 \mathrm{mg}$ administered as an intravenous infusion over $30 \mathrm{~min}$ every 3 weeks until disease progression, unacceptable toxicity, or up to 24 months in patients without disease progression'. This label reflects the 35-dose (2year) maximum duration of pembrolizumab treatment specified by protocol in the two registration trials, KN010 [3] and KN024 [1]. Patients with disease progression after completing the 35 doses were eligible for a second course of pembrolizumab (up to 17 doses/1 year).

The KN024 trial is an open-label randomized study comparing first-line pembrolizumab monotherapy with platinum-based chemotherapy for metastatic NSCLC (stage IV) with PD-L1 TPS $\geq 50 \%$ and no sensitizing $E G F R$ mutation or $A L K$ translocation [1,4]. In KN024, after a median follow-up of 11.2 months as of 9 May 2016, the median pembrolizumab treatment duration was 7.0 months (range: 1 day to 18.7 months), and the median number of doses administered was 10.5 (range: 1-26) [1]. A recent update of KN024 through 10 July 2017, reports median pembrolizumab treatment duration of 7.9 months (range: 1 day to 28.8 months) [4]. A subsequent trial, $\mathrm{KN} 042$, is examining first-line pembrolizumab monotherapy for a patient population with locally advanced or metastatic NSCLC and PD-L1 TPS $\geq 1 \%$, also with no targetable mutations [5]. In KN042, overall, after a median follow-up of 12.8 months (range: $0.1-38.3$ ), patients in the pembrolizumab treatment group had received a median of nine doses (range 1-36) [5].

Future Medicine 
The optimal duration of advanced NSCLC treatment with pembrolizumab and other immune checkpoint inhibitors is a topic of ongoing study in clinical trials. However, most patients with NSCLC receive treatment outside of clinical trials, and the time on treatment with pembrolizumab for advanced NSCLC in the real-world setting is not well-understood. Treatment duration is a key concern for clinicians, as well as for payers because of the budget impact. Moreover, an understanding of treatment duration may be relevant for patients who are interested in requesting treatment-free intervals to determine how well their disease is controlled after coming off therapy.

Some authors have suggested that time on treatment (also called time to treatment discontinuation [TTD]) could serve as a practical surrogate to measure the benefits of therapy, with preliminary evidence suggesting correlations with overall survival (OS) [6] and progression-free survival (PFS) [7]. Indeed, the results of a recently published analysis of 18 randomized clinical trials of patients with metastatic NSCLC indicate that TTD is associated with PFS across therapeutic classes when studied at the patient level [8]. The authors recommend that TTD with continuously administered therapies should be further explored as a pragmatic end point for real-world trials.

The widespread adoption of electronic health records (EHRs) provides an opportunity to study measures such as the time on treatment with systemic anticancer therapies in real-world settings [9-11]. Moreover, the need for research in real-world clinical practice settings is gaining increased attention because of the recognition that the close monitoring and follow-up achievable in randomized controlled trials differs from the ecology of care in clinical practice, where patient populations also tend to be more heterogeneous than highly selected trial populations [12-16].

The objective of our study was to estimate real-world time on treatment (rwToT) with first-line pembrolizumab monotherapy at US oncology clinics in a population with stage IV NSCLC and PD-L1 TPS $\geq 50 \%$. We also calculated pembrolizumab treatment duration using individual patient-level data from KN024 and from the subpopulation of patients with stage IV NSCLC and PD-L1 TPS $\geq 50 \%$ in KN0 $22[4,5,17,18]$.

\section{Methods}

\section{Real world data source \& study population}

This retrospective cohort study utilized the Flatiron Health database, a demographically and geographically diverse database containing longitudinal EHR data of more than 2.1 million patients with cancer and active EHRs seen at over 280 community and academic cancer clinics across the USA [19]. The patient-level EHR data combine structured data, such as test results and prescribed drugs, with unstructured data, such as free-text physicians' notes and pathology reports, collected via technology-enabled chart abstraction.

The Flatiron Health database includes information on demographics, diagnoses (cancer as well as noncancer diagnoses), visits to the cancer facility, laboratory and vital tests and results, disease and biomarker characteristics, Eastern Cooperative Oncology Group performance status (ECOG PS), medication orders and administration details, and mortality. For each medication, drug name(s), route, dose, units and dates are included. Information on systemic treatment, as evidenced by an order or administration of an antineoplastic agent recorded in the EHR, is utilized in establishing oncologist-defined, rule-based line of therapy details [20]. Date of death is identified using Flatiron Health mortality 2.0 [21], a recently validated real-world composite mortality end point that amalgamates structured and unstructured data from EHRs, external commercial sources and the US Social Security Death Index [22].

Patients included in this study were drawn from the Flatiron Health advanced NSCLC cohort, for which eligibility requires at least two documented clinic visits and a pathologically confirmed diagnosis of stage IIIB or IV NSCLC on or after 1 January 2011. The data are refreshed monthly, and the cutoff date for the study dataset was 30 November 2018.

The study inclusion criteria identified patients initiating first-line pembrolizumab monotherapy through May 2018 to allow for $\geq 6$ months of follow-up until database cutoff; patients who died within 6 months but met other eligibility criteria were included. In addition, eligible patients had ECOG PS $0-2$ and stage IV NSCLC, with PD-L1 TPS $\geq 50 \%$, and negative or unknown status for sensitizing EGFR mutation, $A L K$ translocation and ROS1 rearrangement. Patients with no structured activity (defined by visits, medication administrations and orders) within 90 days of the advanced NSCLC diagnosis and those who received first-line systemic anticancer therapy in a clinical trial were excluded. Our analysis primarily relied on medication administration details of pembrolizumab monotherapy when administered in the first-line setting, defined as starting after or $\leq 14$ days before the metastatic NSCLC diagnosis date. 
Approval of the study protocol was obtained through Flatiron procedure and approved by the Copernicus Group Institutional Review Board before study conduct and included a waiver of informed consent. Provisions were in place to prevent re-identification of de-identified data in order to protect patients' confidentiality.

\section{Study end point}

The rwToT was the primary outcome measure, calculated as the (date of the last pembrolizumab dose - date of first pembrolizumab dose) +1 day and defined as the length of time between first and last administration dates before discontinuation of first-line pembrolizumab monotherapy. Patients who had a record of initiating the next line of therapy, or who died while receiving first-line pembrolizumab monotherapy, were considered discontinued at their last pembrolizumab administration date. If none of these events were identified, then having a gap of $\geq 120$ days between the last administration date of first-line pembrolizumab monotherapy and last known activity date in the dataset was considered discontinued at the last administration date. If none of the discontinuation criteria were met, patients were considered censored at their last administration date. The definition of this rwToT outcome measure is the same as the definition of TTD used in the Friends of Cancer Research white paper [6] and the recently published study of Blumenthal et al. [8].

\section{Clinical trial data sources}

To provide context to rwToT results, we also conducted a post hoc analysis of KEYNOTE trial data, using data on file, to describe pembrolizumab treatment duration in two randomized, open-label trials of first-line pembrolizumab monotherapy for NSCLC, restricting the analyses to patients with metastatic NSCLC with PD-L1 TPS $\geq 50 \%[4,17,18]$. The KN024 trial was conducted at 142 sites in 16 countries including the USA [4], and the KN042 trial was conducted at 213 sites in 32 non-US countries [5]. All patients in the pembrolizumab treatment group of KN024 were included ( $\mathrm{n}=154$ ), as stage IV NSCLC and PD-L1 TPS $\geq 50 \%$ were inclusion criteria. For the KN042 trial, which included patients with stage III NSCLC and PD-L1 TPS $\geq 1 \%$, we restricted the analyses to those with PD-L1 TPS $\geq 50 \%$ and stage IV diagnosis $(\mathrm{n}=271)$. The KN024 cutoff date was 10 July 2017, and the KN042 cutoff date was 26 February 2018.

Pembrolizumab treatment duration in the KEYNOTE trials was calculated similarly to rwToT as the (date of the last pembrolizumab dose - date of pembrolizumab first dose) +1 day; patients were censored at last dose date if still on therapy at the time of trial cutoff.

\section{Statistical analyses}

Descriptive analyses were conducted for patient demographic and baseline clinical characteristics from Flatiron Health data as well as patient and disease characteristics from the KEYNOTE trials (for KN042, limited to the patient subpopulation with stage IV NSCLC and PD-L1 TPS $\geq 50 \%)[4,5,18]$. We determined frequencies and percentages for categorical variables and mean (standard deviation) or median (range) for variables measured on the continuous or interval scale. Analyses of the Flatiron Health data were stratified by ECOG PS $0-1$ and ECOG PS 2.

The Kaplan-Meier (KM) time-to-event method was used to estimate median rwToT and the restricted mean rwToT, as well as the percentage of patients still receiving pembrolizumab at 6,12, 18 and 24 months [23,24]. Data at 18 and 24 months were considered not sufficiently mature, using the Pocock 10\% rule as guidance [25]. Therefore, for these time points, the restricted mean rwToT was extrapolated using parametric functions that were fitted to the KM data. The best-fitting parametric function was selected from exponential, Weibull, log-normal, loglogistic, Gompertz and generalized gamma distributions. Statistical tests based on the Akaike information criterion, combined with visual inspection (comparing fitted distribution to study $\mathrm{KM}$ plots to assess closeness of fit overall), were used to select the best-fitting parametric distributions. The curve fitting was carried out in line with NICE Decision Support Unit guidelines [26]. In addition, as further validation, we calculated the restricted mean at 18 and 24 months based on extrapolation using a proportional hazards model with trial reference (KN024) for the ECOG PS 0-1 stratum. As a sensitivity analysis, the KM estimation was also conducted for rwToT and on-treatment rates restricted to patients with known negative status for sensitizing $E G F R$ mutation and $A L K$ translocation. The distribution of number of pembrolizumab doses was also examined.

For the KEYNOTE trials, we similarly calculated KM median and restricted mean duration of treatment and on-treatment rates at 6, 12, 18 and 24 months using patient-level data on file [17,18]. An overlay of the KM plots 


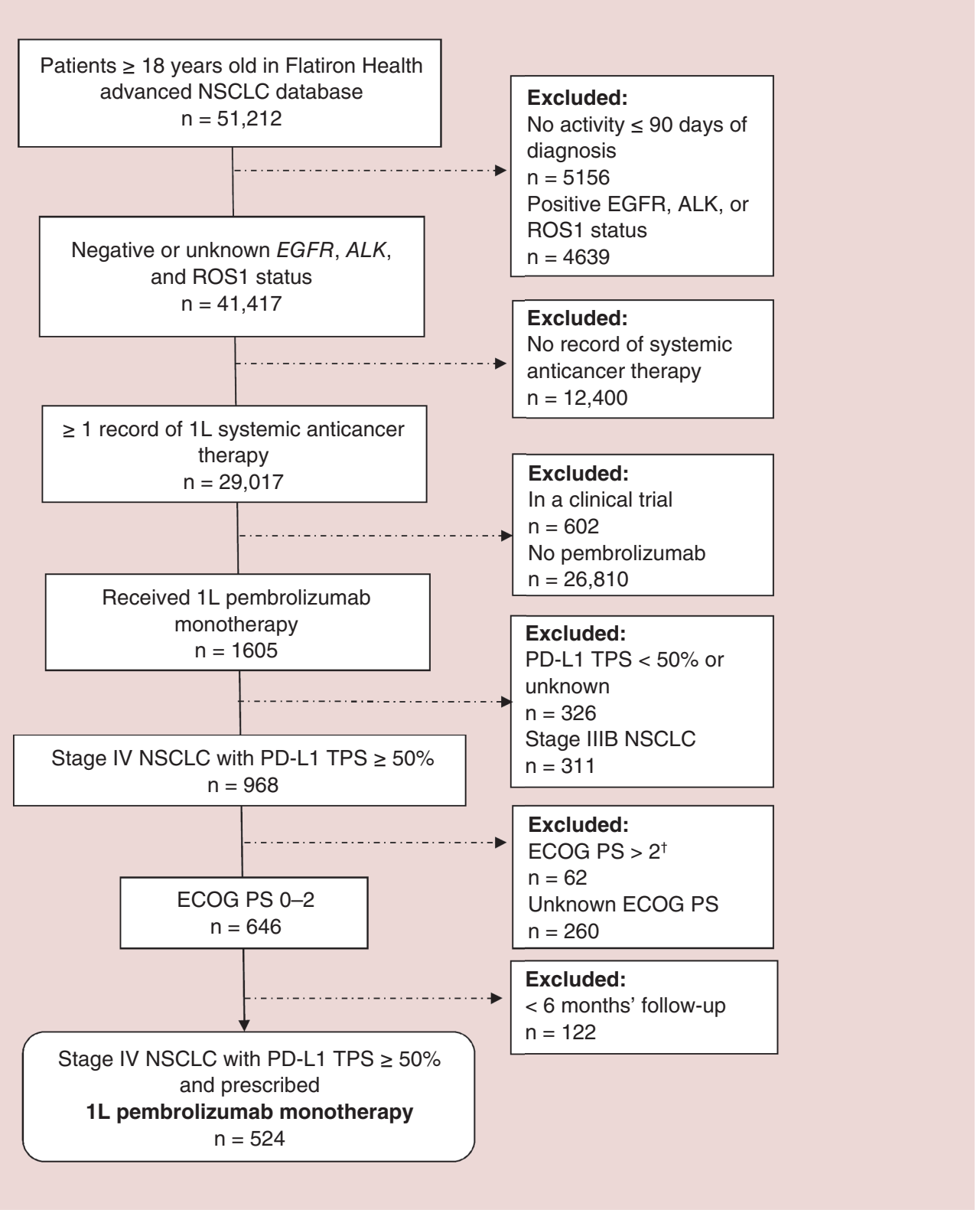

Figure 1. Selection of eligible patients in the database.

†59 excluded patients had ECOG PS of 3, and 3 excluded patients had ECOG PS of 4.

1L: First-line therapy; ALK: Anaplastic lymphoma kinase translocation; ECOG PS: Eastern Cooperative Oncology Group performance status; EGFR: Sensitizing epidermal growth factor receptor mutation; PD-L1: Programmed death-ligand 1; TPS: Tumor proportion score.

of the KN024 and KN042 treatment durations together with the Flatiron rwToT in the ECOG PS 0-1 stratum was also generated.

Analyses were carried out using the $\mathrm{R}$ language for statistical computing (version 3.5.0; R Core Team, 2018). Flatiron Health, Inc. did not participate in the analysis of the data.

\section{Results}

Patients \& follow-up duration

Of 1605 patients prescribed first-line pembrolizumab monotherapy for advanced NSCLC, 1279 (80\%) had PD-L1 TPS $\geq 50 \%$, and, of those, 968 (76\%) had stage IV NSCLC at diagnosis (Figure 1). Among them we identified a total of 524 eligible patients with minimum 6 months' follow-up (until database cutoff) and known ECOG PS 
Table 1. Baseline demographic and clinical characteristics of patients with stage IV non-small-cell lung cancer with PD-L1 tumor proportion score $\geq 50 \%$, without targetable mutations.

\begin{tabular}{|c|c|c|c|c|}
\hline \multirow[t]{2}{*}{ Variable } & \multicolumn{2}{|c|}{ Real-world EHR data } & \multicolumn{2}{|c|}{ Clinical trial data $[4,17,18]$} \\
\hline & ECOG PS 0-1 $(n=386)$ & ECOG PS $2(n=138)$ & KN024 $(n=154)$ & $\begin{array}{l}\text { KN042 subpopulation } \\
(n=271)\end{array}$ \\
\hline Male sex & $210(54.4)$ & $64(46.4)$ & $92(59.7)$ & $184(67.9)$ \\
\hline Age, median (range), years: & $72(45-85)$ & $73(44-85)$ & $64.5(33-90)$ & $63(25-89)$ \\
\hline$-\geq 65$ years & $274(71.0)$ & $105(76.1)$ & $77(50.0)$ & $117(43.2)$ \\
\hline Race data available: & 347 (89.9) & $121(87.7)$ & $152(98.7)$ & $271(100)$ \\
\hline - White ${ }^{\dagger}$ & $266(76.7)$ & $83(68.6)$ & $125(82.2)$ & $168(62.0)$ \\
\hline - Black ${ }^{\dagger}$ & $31(8.9)$ & $20(16.5)$ & $2(1.3)$ & 0 \\
\hline - Asian ${ }^{\dagger}$ & $13(3.7)$ & $3(2.5)$ & $25(16.4)$ & $86(31.7)$ \\
\hline - Other race ${ }^{\dagger}$ & $37(10.7)$ & $15(12.4)$ & 0 & $17(6.3)$ \\
\hline Current/former smoker: & $356(92.2)$ & $131(94.9)$ & $149(96.8)$ & $213(78.6)$ \\
\hline - No smoking history & $30(7.8)$ & $6(4.3)^{\ddagger}$ & $5(3.2)$ & $58(21.4)$ \\
\hline $\mathrm{CCl}$ score, mean (SD): & $3.2(3.1)$ & $3.5(3.2)$ & - & - \\
\hline - Range & $0-10$ & $0-10$ & - & - \\
\hline \multicolumn{5}{|l|}{ Histology: } \\
\hline - Nonsquamous & $269(69.7)$ & $101(73.2)$ & $125(81.2)$ & $182(67.2)$ \\
\hline - NSCLC histology NOS & $19(4.9)$ & $7(5.1)$ & 0 & 0 \\
\hline - Squamous & $98(25.4)$ & $30(21.7)$ & $29(18.8)$ & $89(32.8)$ \\
\hline ECOG performance status of 1 & $246(63.7)$ & 0 & $99(64.3)^{\S}$ & $187(69.0)$ \\
\hline Brain metastases $\mathbb{I}$ & $47(12.2)$ & $13(9.4)$ & $18(11.7)$ & $19(7.0)$ \\
\hline \multicolumn{5}{|c|}{$\begin{array}{l}\text { Data are } \mathrm{n}(\%) \text { unless otherwise indicated. } \\
\text { †Percentages refer to those with available data. } \\
\text { ¥Smoking history was not recorded for one patient in the ECOG PS } 2 \text { cohort. } \\
\text { \$One patient enrolled in KNO24 had ECOG PS of } 2 \text {. } \\
\mathbb{I} \text { In KNO24/KNO42, brain metastases were pretreated. Information about pretreatment was not available for real-world EHR data. } \\
\text { CCI: Charlson comorbidity index; ECOG PS: Eastern Cooperative Oncology Group performance status; EHR: Electronic health record; KN: KEYNOTE; NSCLC histology NOS } \\
\text { Non-small-cell lung cancer histology not otherwise specified. }\end{array}$} \\
\hline
\end{tabular}

of 0-2, including 386 (74\%) with ECOG PS 0-1 and 138 (26\%) with ECOG PS 2. As of 30 November 2018, the median duration of observed follow-up from pembrolizumab initiation to database cutoff was 16.0 (range: 6.0-37.3) and 16.5 months (range: 6.2-36.5) for ECOG PS 0-1 and ECOG PS 2 cohorts, respectively. All but seven patients overall were treated at community (rather than academic) cancer clinics.

The median ages in the ECOG PS $0-1$ and ECOG PS 2 cohorts were similar (72 and 73 years, respectively; Table 1), with 149 patients (39\%) and $64(46 \%)$ aged 75 years or older, respectively. The ECOG PS $0-1$ cohort included higher proportions of men ( 54 vs $46 \%$, respectively) and of white patients ( 77 vs $69 \%$, respectively) than the ECOG PS 2 cohort. The majority of patients in each real-world cohort $(\geq 92 \%)$ had a positive smoking history, and almost three-quarters of patients in each cohort had nonsquamous NSCLC (Table 1). A total of 299 patients (77\%) in the ECOG PS 0-1 cohort and 103 patients (75\%) in the ECOG PS 2 cohort had tumors that tested negative for both sensitizing $E G F R$ mutation and $A L K$ translocation; the other patients' tumors were not tested or had unknown status.

We made no statistical comparisons among the cohorts; however, the ages of patients in the trial-eligible ECOG PS 0-1 real-world cohort (median age, 72 years) tended to be older than those in KN024 (median age, 65 years) and KN042 subpopulation with stage IV NSCLC, PD-L1 TPS $\geq 50 \%$ (median age, 63 years; Table 1); and the proportions of men in the real-world cohort tended to be smaller than in the clinical trial cohorts $(54 \%$ men vs $60-68 \%$ in KN024/042). We observed similar smoking history and histology distribution in both of the real-world and the KN024 patient cohorts, while in KN042 there were lower proportions of current/former smokers and greater proportions of patients with squamous histology than in the other cohorts (Table 1).

Time on treatment with first-line pembrolizumab monotherapy in real-world \& clinical trial settings As of 30 November 2018, 61\% of patients in the ECOG PS 0-1 cohort and 79\% patients in the ECOG PS 2 cohort had discontinued pembrolizumab monotherapy (Table 2). Pembrolizumab rwToT was markedly longer for 
Table 2. Time on treatment with first-line pembrolizumab monotherapy in real-world (rwToT) and clinical trial (ToT) settings calculated using the Kaplan-Meier method for patients with stage IV non-small-cell lung cancer, PD-L1 tumor proportion score $\geq 50 \%$, without targetable mutations.

\begin{tabular}{|c|c|c|c|c|}
\hline \multirow[t]{2}{*}{ Variable } & \multicolumn{2}{|c|}{ Real-world EHR data ${ }^{\dagger}$} & \multicolumn{2}{|c|}{ Clinical trial data $\ddagger$} \\
\hline & ECOG PS 0-1 $(n=386)$ & ECOG PS $2(n=138)$ & KN024 $(n=154)$ & $\begin{array}{l}\text { KN042 subpopulation } \\
(\mathrm{n}=271)\end{array}$ \\
\hline Discontinued, n (\%) & $237(61.4)$ & $109(79.0)$ & $131(85.1)$ & $227(83.8)$ \\
\hline Observed follow-up, median (range), months ${ }^{\S}$ & $16.0(6.0-37.3)$ & $16.5(6.2-36.5)$ & $25.0(20.4-33.7)$ & $23.1(12.0-37.6)$ \\
\hline Patient follow-up, median (range), months ${ }^{\S}$ & $10.9(0.1-37.3)$ & 5.2 (1 day-23.6) & $22.0(0.1-32.9)$ & $14.0(0.1-37.2)$ \\
\hline rwToT/ToT, I median ( $95 \% \mathrm{Cl})$, months & $6.9(5.6-8.3)$ & $2.3(1.4-3.5)$ & $7.9(6.2-11.7)$ & $6.6(4.9-8.5)$ \\
\hline \multicolumn{5}{|l|}{ Restricted mean rwToT/ToT $\mathbb{I}(95 \% \mathrm{Cl})$, months: } \\
\hline - Restricted to 6 months & $4.2(4.0-4.5)$ & $2.8(2.4-3.2)$ & $4.4(4.0-4.7)$ & $4.2(3.9-4.4)$ \\
\hline - Restricted to 12 months & $6.9(6.4-7.3)$ & $4.3(3.5-5.0)$ & $7.2(6.5-8.0)$ & $6.8(6.2-7.3)$ \\
\hline $\begin{array}{l}\text { - Restricted to } 18 \text { months, est. by parametric } \\
\text { function/ToT }\end{array}$ & 8.9 (8.0-9.9) (Weibull) & 5.2 (3.7-6.5) (LogNorm) & $9.3(8.2-10.4)$ & $8.8(7.9-9.6)$ \\
\hline \multicolumn{5}{|l|}{ - Restricted to 24 months } \\
\hline - rwToT est. by parametric function/ToT & 10.5 (9.4-11.7) (Weibull) & $6.1(4.3-8.0)$ (LogNorm) & $11.0(9.5-12.5)$ & $10.4(9.3-11.5)$ \\
\hline - rwToT by trial-based extrapolation & $10.5(9.7-11.4)$ & - & - & - \\
\hline \multicolumn{5}{|l|}{ On-treatment rate, $\%(95 \% \mathrm{Cl})$ : } \\
\hline At 6 months & $53.7(48.5-58.6)$ & $30.2(22.6-38.1)$ & $58.4(50.2-65.8)$ & $52.0(45.9-57.8)$ \\
\hline At 12 months & $36.4(31.2-41.6)$ & $16.2(9.8-24.0)$ & $40.3(32.5-47.9)$ & $37.6(31.9-43.4)$ \\
\hline At 18 months & $31.7(26.3-37.3)$ & $12.5(6.4-20.6)$ & $29.9(22.9-37.2)$ & $30.1(24.5-35.8)$ \\
\hline At 24 months & NR & NR & $12.7(7.2-19.8)$ & $6.6(3.4-11.4)$ \\
\hline \multicolumn{5}{|c|}{ 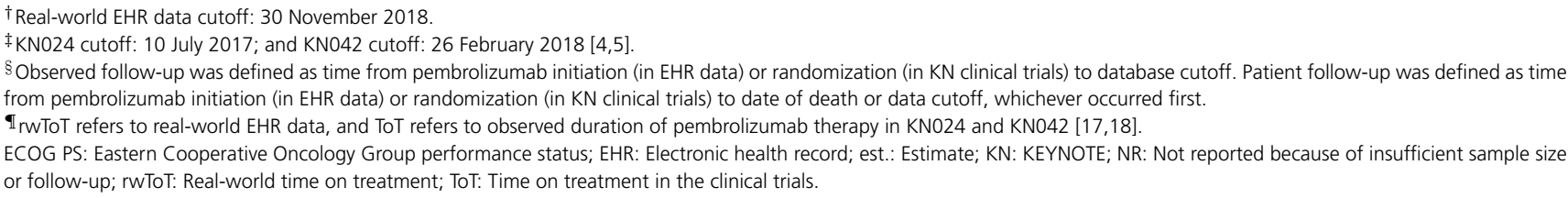 } \\
\hline
\end{tabular}

Table 3. Number of pembrolizumab doses received by patients in the two real-world electronic health record cohorts.

\begin{tabular}{|lll|}
\hline Doses $(\mathbf{n})$ & ECOG 0-1 $(\mathbf{n}=386)$ & ECOG $2(\mathbf{n}=138)$ \\
\hline Median (range) & $9(1-35)$ & $3(1-33)$ \\
\hline 1 & $39(10.1)$ & $41(29.7)$ \\
\hline 2 & $22(5.7)$ & $16(11.6)$ \\
\hline 3 & $29(7.5)$ & $13(9.4)$ \\
\hline 4 & $35(9.1)$ & $6(4.3)$ \\
\hline 5 & $19(4.9)$ & $10(7.2)$ \\
\hline$\geq 6$ & $242(62.7)$ & $52(37.7)$ \\
\hline Data are $n(\%)$ unless otherwise indicated. & & \\
ECOG: Eastern Cooperative Oncology Group. & & \\
\hline
\end{tabular}

patients with ECOG PS 0-1 (median of 6.9 months; 95\% CI: 5.6-8.3) than for those with ECOG PS 2 (median of 2.3 months; 95\% CI: 1.4-3.5; Figure 2). No patient had yet received 24 months of therapy. The restricted mean rwToT and on-treatment rates at the landmark time points followed a similar pattern, with estimates for the ECOG PS 0-1 cohort substantially better than those in the ECOG PS 2 cohort (Table 2). In the ECOG PS 0-1 cohort, $10 \%$ of patients received only one pembrolizumab dose (median, nine doses) as compared with $30 \%$ who received only one dose in the ECOG PS 2 cohort (median, three doses; Table 3).

For the ECOG PS 0-1 cohort, we observed that the median rwTOT of 6.9 months approached the median duration of pembrolizumab treatment recorded in KN024 (7.9 months; 95\% CI: 6.2-11.7) and was similar to that in KN042 subpopulation with stage IV NSCLC, PD-L1 TPS $\geq 50 \%$ (6.6 months; 95\% CI: 4.9-8.5; Figure 3). Moreover, the restricted mean rwToTs at $6,12,18$ and 24 months for patients with ECOG PS 0-1 were similar to those estimated in the clinical trials (Table 2). At 12 months, the restricted mean rwToT for patients with ECOG 


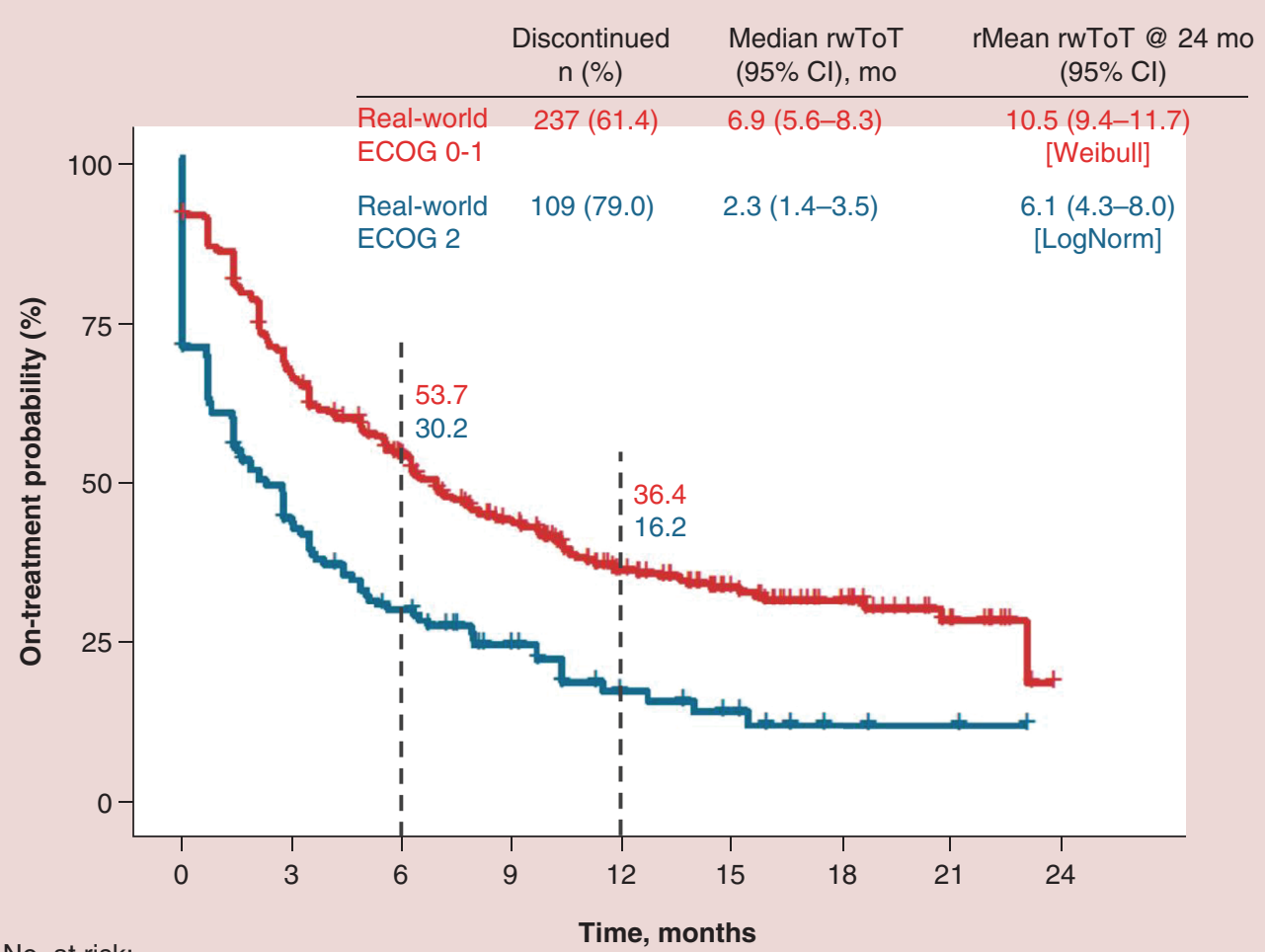

No. at risk:

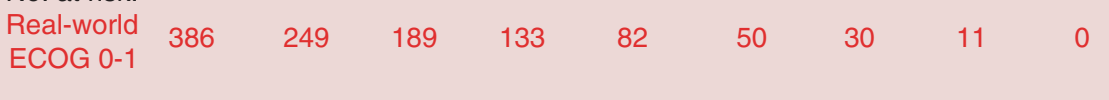

$\begin{array}{llllllllll}\text { Real-world } & 138 & 57 & 37 & 24 & 12 & 8 & 3 & 2 & 0\end{array}$

ECOG 2

Figure 2. Real-world time on treatment (rwToT). Kaplan-Meier plot depicting rwToT with pembrolizumab for patients with stage IV non-small-cell lung cancer, PD-L1 tumor proportion score $\geq 50 \%$ and Eastern Cooperative Oncology Group performance status of $0-1$ and 2.

rMean: Restricted mean.

PS 0-1 was 6.9 months (95\% CI: 6.4-7.3) as compared with 12-month restricted mean treatment durations of 7.2 (95\% CI: 6.5-8.0) and 6.8 months (95\% CI: 6.2-7.3) in KN024 and KN042, respectively. Similarly, the restricted mean rwToT estimated at 24 months for patients with ECOG PS 0-1 (10.5 months; 95\% CI: 9.4-11.7; Weibull distribution) resembled the 24-month restricted means for duration of pembrolizumab treatment in the clinical trials of 11.0 months (95\% CI: 9.5-12.5) and 10.4 months (95\% CI: 9.3-11.5) in KN024 and KN042, respectively. Results were similar when validation was done using trial-based extrapolation for patients with ECOG PS 0-1 (Table 2).

The on-treatment rates for the ECOG PS $0-1$ cohort were also very similar to those for the clinical trial populations (Table 2) estimated at 36.4\% (95\% CI: 31.2-41.6\%) at 12 months and 31.7\% (95\% CI: $26.3-$ $37.3 \%)$ at 18 months. The on-treatment rates at 24 months were not estimated because of limited data maturity.

\section{Sensitivity analysis: NSCLC with known negative status for sensitizing EGFR mutation \& ALK translocation}

For the subpopulation of patients with NSCLC with known negative status for $E G F R / A L K$ aberrations, the results of KM analyses of rwToT were similar to those for the full cohort among patients with ECOG PS 0-1 (median 6.5 months) and slightly shorter for those with ECOG PS 2 (median 1.9; Table 4). All values for the restricted mean rwToTs and on-treatment rates at 6,12 and 18 months in both ECOG PS cohorts were similar to those for the full ECOG PS cohorts, for example, the estimated restricted mean rwToT at 24 months in the ECOG PS 0-1 cohort was 10.4 months (95\% CI: 9.2-11.8), and that in the ECOG PS 2 cohort was 5.9 months (95\% CI: 3.8-8.0; Table 4). The pembrolizumab dose distributions were the same as for the full cohorts. 


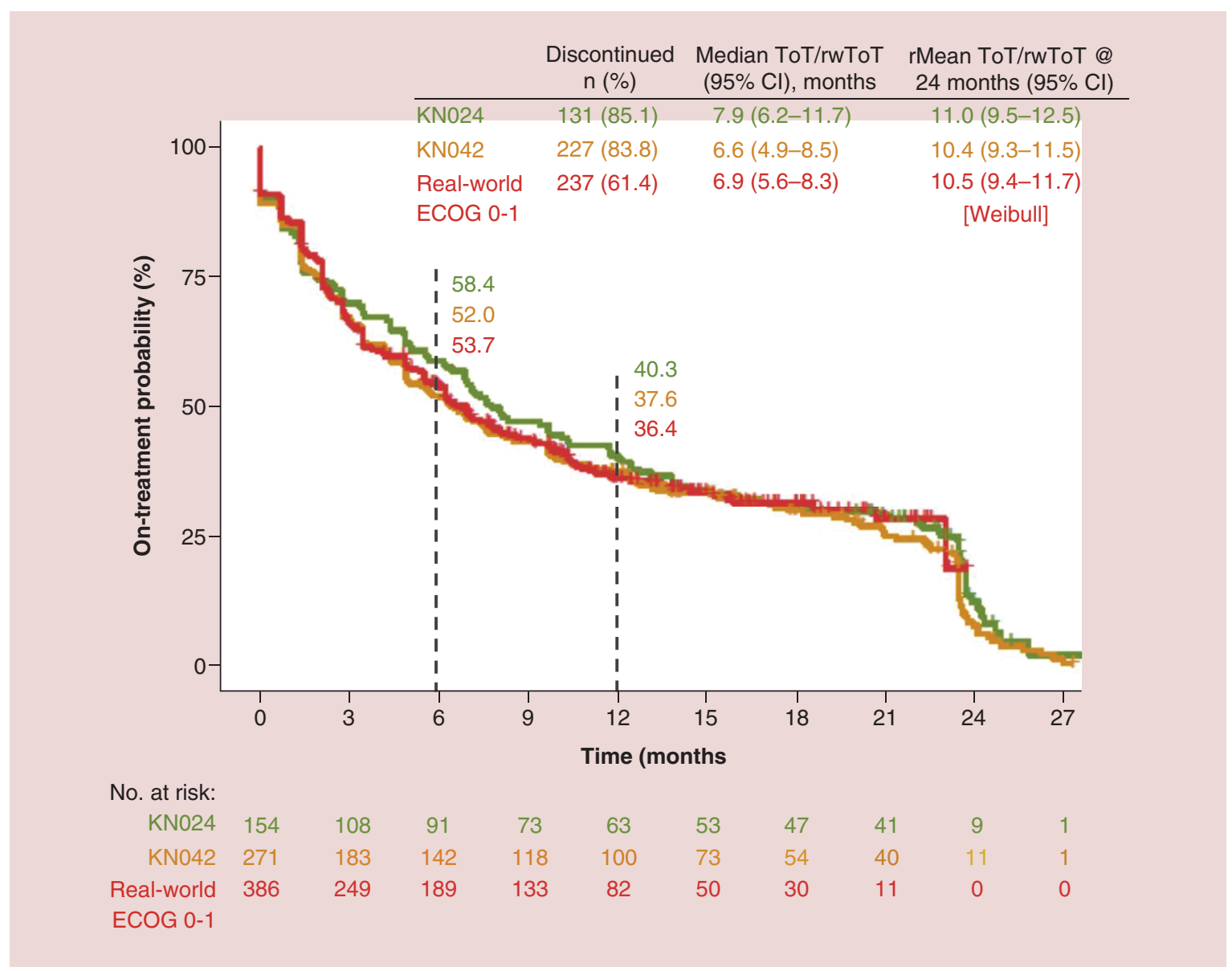

Figure 3. Real-world time on treatment (rwToT) and clinical trial treatment duration. Kaplan-Meier plot depicting rwTOT with pembrolizumab for patients with stage IV NSCLC, PD-L1 TPS $\geq 50 \%$ and ECOG performance status of 0-1 and pembrolizumab treatment duration for clinical trial patients in KEYNOTE-024 and KEYNOTE-042 (subpopulation with stage IV NSCLC, PD-L1 TPS $\geq 50 \%$ ).

rwToT refers to real-world data, and ToT refers to observed duration of pembrolizumab therapy in the clinical trials $[17,18]$.

ECOG: Eastern Cooperative Oncology Group; NSCLC: Non-small-cell lung cancer; rMean: Restricted mean.

\section{Discussion}

For the trial-eligible population (with ECOG PS 0-1) in this retrospective cohort study, we observed that rwToT and on-treatment rates with first-line pembrolizumab monotherapy for metastatic NSCLC with PD-L1 TPS $\geq 50 \%$ and negative or unknown $E G F R / A L K$ aberration status were similar to those for patients with metastatic NSCLC with PD-L1 TPS $\geq 50 \%$ enrolled in the KN024 and KN042 trials. These findings were supported by results of sensitivity analyses restricted to the subgroup of patients with known negative status for sensitizing $E G F R$ mutation and $A L K$ translocation. Instead, for real-world patients who would have been excluded from the KEYNOTE trials (with ECOG PS 2), the pembrolizumab rwToT was substantially shorter and on-treatment rates substantially lower. Almost a third of patients in the ECOG PS 2 cohort received only one dose of pembrolizumab.

The finding of shorter rwToT for patients with ECOG PS 2 was not surprising, as performance status, despite being a subjective measure, is an important prognostic factor in metastatic NSCLC. A recent review found that lower ECOG PS score (or higher Karnofsky performance status score) was a prognostic factor for better outcomes across multiple studies and different patient populations with advanced NSCLC [27].

Our study included patients treated with first-line pembrolizumab monotherapy in the setting of US oncology clinics, excluding any patients treated in clinical trials. We found that the real-world ECOG PS 0-1 cohort was older, on average, and included a numerically higher percentage of women than the pembrolizumab treatment groups in KN024 and KN042 subpopulation with metastatic NSCLC with PD-L1 TPS $\geq 50 \%[4,18]$. These 
Table 4. Real-world time on treatment with first-line pembrolizumab monotherapy, calculated using the Kaplan-Meier method for patients with stage IV non-small-cell lung cancer, PD-L1 tumor proportion score $\geq 50 \%$ : sensitivity analysis including only those with known negative status for sensitizing EGFR mutation and ALK translocation.

\begin{tabular}{|c|c|c|}
\hline \multirow[t]{2}{*}{ Variable } & \multicolumn{2}{|c|}{ Real-world EHR data ${ }^{\dagger}$} \\
\hline & ECOG PS 0-1 $(n=299)$ & ECOG PS $2(n=103)$ \\
\hline Discontinued, n (\%) & 185 (61.9) & $82(79.6)$ \\
\hline Observed follow-up, median (range) ${ }^{\ddagger}$ & $16.9(7-38.3)$ & $17.7(7.2-37.5)$ \\
\hline Patient follow-up, median (range) $)^{\ddagger}$ & $12.4(0.1-38.3)$ & 3.5 (1 day-24.6) \\
\hline rwToT, median $(95 \% \mathrm{Cl})$, months & $6.5(5.6-8.5)$ & $1.9(0.8-3.0)$ \\
\hline \multicolumn{3}{|l|}{ Restricted mean rwToT $(95 \% \mathrm{Cl})$, months: } \\
\hline - Restricted to 6 months & $4.3(4.0-4.5)$ & $2.7(2.2-3.2)$ \\
\hline - Restricted to 12 months & $6.9(6.4-7.4)$ & $4.1(3.2-5.0)$ \\
\hline - Restricted to 18 months, est. by parametric function & 8.9 (7.9-10.0) (Weibull) & $5.0(3.3-6.5)$ (LogNorm) \\
\hline \multicolumn{3}{|l|}{ - Restricted to 24 months } \\
\hline - rwToT est. by parametric function & 10.4 (9.2-11.8) (Weibull) & $5.9(3.8-8.0)$ (LogNorm) \\
\hline - rwToT by trial-based extrapolation & $10.5(9.6-11.4)$ & - \\
\hline \multicolumn{3}{|l|}{ On-treatment rate, $\%(95 \% \mathrm{Cl})$} \\
\hline At 6 months & $51.7(45.8-57.3)$ & $29.5(20.8-38.7)$ \\
\hline At 12 months & $34.5(28.6-40.4)$ & $16.1(8.9-25.3)$ \\
\hline At 18 months & $31.9(25.8-38.2)$ & $10.8(4.3-20.6)$ \\
\hline At 24 months & NR & NR \\
\hline \multicolumn{3}{|c|}{$\begin{array}{l}\dagger \text { Real-world EHR data cut-off: } 30 \text { November } 2018 \text {. } \\
\text { ¥Observed follow-up was defined as the duration of EHR database follow-up from pembrolizumab initiation to database cutoff. Patient follow-up was defined as time from } \\
\text { pembrolizumab initiation in EHR data to the date of death or data cutoff, whichever occurred first. } \\
\text { ECOG PS: Eastern Cooperative Oncology Group performance status; EHR: Electronic health record; est.: Estimate; NR: Not reported because of insufficient sample size or } \\
\text { follow-up. }\end{array}$} \\
\hline
\end{tabular}

discrepancies accorded with the findings of an earlier study that women and the elderly were under-represented among patients participating in clinical trials sponsored by the US National Cancer Institute in 2000 through 2002 [14]. That study also found discrepancies by race, with blacks being less likely to enroll in clinical trials. In our study, black patients numbered 31 (9\%) and 20 (17\%) in ECOG PS 0-1 and ECOG PS 2 cohorts, although we note that a comparison with the racial make-up of KN024 and KN042 would be not be meaningful because those trials were conducted primarily at sites in countries other than the USA.

Our study population was identified using the KEYNOTE trial eligibility criteria; however, there were some inherent differences from the trial populations. Because we drew on EHR data, it was not possible to identify patients with life expectancy of at least 3 months, as required by KN024 and KN042 [1,5]. Moreover, we did not restrict study eligibility to NSCLC with known negative status for $E G F R / A L K$ aberrations, although the majority of patients in each ECOG PS cohort fulfilled this criterion. As noted above, there were differences from the KEYNOTE trials in sites of care. Specifically, in our study population, $>98 \%$ of patients were treated at community practices in the USA, while KN024 recruited primarily from academic medical centers in the USA, Canada, Western Europe, Israel, and Japan, and the KN042 sites were located primarily in Eastern Europe, Latin America, and Asia Pacific, with no sites in the USA [1,5]. Differences have been reported between academic and nonacademic hospitals with regard to treatment patterns and survival for patients with NSCLC [28].

An important study limitation is the retrospective assessment of performance status from EHR data. Moreover, patients with unknown ECOG PS comprised a substantial proportion of patients in the dataset and were excluded. The generalizability of our findings is limited to real-world patients with metastatic NSCLC with PD-L1 $\geq 50 \%$, as per our eligibility criteria. Furthermore, we were not able to evaluate the reasons for therapy discontinuation, nor patient outcomes with pembrolizumab therapy. Future studies are needed to examine additional outcomes such as PFS and OS associated with first-line pembrolizumab monotherapy for NSCLC in real-world settings.

Strengths of this study include the use of a well-regarded EHR-derived database [19] and a well-characterized patient population. The majority of study patients received treatment in community clinics, where most patients with metastatic NSCLC are treated in the USA. We followed a large study population for a length of time sufficient 
to determine rwToT up to the 12-month restricted mean with adequate sample size. The KM method was used to account for patients who were still on pembrolizumab therapy at the time of database cutoff. We were able to estimate this end point using comparable methods and individual patient-level data from both real-world and clinical trials to accurately estimate the benefit of pembrolizumab in the real-world setting. In addition, because of the labeled dosage for pembrolizumab therapy up to 24 months in patients without disease progression, we estimated the restricted mean at 24 months via extrapolation using the best-fitting parametric function.

Our definition of rwToT is aligned with TTD as defined by the Friends of Cancer in the proposed framework to evaluate real-world end points [6] and subsequently employed by Blumenthal and coworkers [8] in their analysis of NSCLC clinical trials submitted to the US FDA. They suggest that TTD could serve as a pragmatic end point in real-world settings to assess both safety and efficacy of anticancer therapies that are commonly administered beyond objective disease progression per RECIST criteria [8,29], as may be the case with pembrolizumab. In their study, there was also a moderate correlation for immune checkpoint inhibitor therapy and OS. Our rwToT findings thus contribute important, albeit preliminary, information to the evidence base for understanding pembrolizumab effectiveness and safety in the real-world setting of US community oncology clinics.

Additional investigations of pembrolizumab rwToT are needed for other NSCLC indications, including pembrolizumab in other lines of therapy, pembrolizumab in combination with chemotherapy, and second-line pembrolizumab monotherapy for NSCLC with PD-L1 TPS 1-49\%. Moreover, with longer follow-up in real-world settings, the extent of re-initiation of pembrolizumab monotherapy and the duration of second courses of pembrolizumab therapy are other parameters of interest. Such studies would also benefit from understanding the reasons for first course discontinuation and the patients' response status at that time. These are topics that are under investigation in the $\mathrm{KN} 010, \mathrm{KN} 024$ and $\mathrm{KN} 042$ trials, which are following patients experiencing complete response who discontinue pembrolizumab and those with disease progression reinitiating pembrolizumab monotherapy for a second course (up to 17 cycles) after completing 35 cycles [4,5,30]. In updated analyses of the KN010 trial, 75 of 79 patients (95\%) with previously treated NSCLC and PD-L1 TPS $\geq 1 \%$ who then received 35 doses ( 2 years) of pembrolizumab therapy had a complete or partial response, and 11 of 14 patients receiving a second course of pembrolizumab had at least a partial response [30]. Results of the follow-up phase of the CheckMate 153 trial, which is comparing continuous vs 1-year fixed duration nivolumab therapy for advanced NSCLC, have not been published as of this writing [31,32]. Another area of study is the continuation of pembrolizumab therapy beyond progression with second-line treatment that is being evaluated in the upcoming ECOG 5163 trial (ClinicalTrials.gov Identifier: NCT03793179) [33]. Finally, there is a need for prognostic and predictive biomarkers to guide the optimal duration of anti-PD-1 therapy for NSCLC, a question recently raised with regard to advanced melanoma therapy [34].

\section{Conclusion}

The results of this study indicate that the real-world duration of first-line pembrolizumab monotherapy is similar to that in the clinical trial setting for patients treated in US community oncology clinics for metastatic NSCLC with PD-L1 TPS $\geq 50 \%$ and negative or unknown $E G F R / A L K$ aberration status who have trial-eligible performance status (ECOG PS 0-1). Instead the real-world duration of first-line pembrolizumab monotherapy is shorter for patients with ECOG PS 2, a population commonly excluded from clinical trials. Our findings suggest that these real-world patients may be experiencing similar outcomes with regard to disease progression and unacceptable toxicity. Patient outcomes and the reasons for pembrolizumab discontinuation remain topics for further study.

\section{Author contributions}

Conception and design of the study: $\bigvee$ Velcheti, S Chandwani and T Burke. Data analysis: X Chen. Interpretation of findings, critical review and revision of the manuscript: $V$ Velcheti, S Chandwani, X Chen, MC Pietanza, T Burke. All authors read and approved the final manuscript.

\section{Acknowledgments}

The authors gratefully acknowledge the help of S Surati, MPH (Merck \& Co., Inc., Kenilworth, NJ, USA) for providing administrative support and S Ting and BL Koch (Merck \& Co., Inc., Kenilworth, NJ, USA) for providing statistical programming support. 
by Merck, participated in the data interpretation and writing of the manuscript. $V$ Velcheti has served in an advisory/consultant role for Merck \& Co., Inc., Kenilworth, NJ, USA; Bristol-Myers Squibb; Genentech; AstraZeneca; Celgene; Novartis; Amgen; Fulgent Genetics; Reddy Labs; Alkermes; Nektar Therapeutics; Foundation Medicine. S Chandwani, X Chen, MC Pietanza and T Burke are employees of Merck Sharp \& Dohme Corp., a subsidiary of Merck \& Co., Inc., Kenilworth, NJ, USA, and stockholders of Merck \& Co., Inc., Kenilworth, NJ, USA. The authors have no other relevant affiliations or financial involvement with any organization or entity with a financial interest in or financial conflict with the subject matter or materials discussed in the manuscript apart from those disclosed.

Medical writing and editorial assistance was provided by EV Hillyer, DVM. This assistance was funded by Merck Sharp \& Dohme Corp., a subsidiary of Merck \& Co., Inc., Kenilworth, NJ, USA.

\section{Ethical conduct of research}

The de-identified dataset supporting the conclusions of this article was provided by Flatiron Health. Institutional Review Board (IRB) approval of the study protocol was obtained through Flatiron procedure and approved by Copernicus Group IRB before study conduct and included a waiver of informed consent. Provisions were in place to prevent re-identification of de-identified data in order to protect patients' confidentiality.

\section{Open access}

This work is licensed under the Attribution-NonCommercial-NoDerivatives 4.0 Unported License. To view a copy of this license, visit http://creativecommons.org/licenses/by-nc-nd/4.0/

\section{Summary points}

- The currently labeled pembrolizumab dosage for treatment of non-small-cell lung cancer (NSCLC) is $200 \mathrm{mg}$ intravenously every 3 weeks for up to 24 months (35 doses); however, the optimal duration of advanced NSCLC treatment with pembrolizumab and other immune checkpoint inhibitors is a topic of ongoing study in clinical trials.

- The duration of pembrolizumab treatment administered outside of clinical trials in real-world settings, where most patients with metastatic NSCLC are treated, is not well-understood.

- Preliminary evidence from randomized NSCLC trials suggests that the time from treatment initiation to treatment discontinuation (or death) for continuously administered therapies (such as pembrolizumab) is associated with progression-free survival and could serve as a pragmatic end point to study NSCLC therapy in real-world settings.

- The objective of our study was to estimate real-world time on treatment (rwToT) with first-line pembrolizumab monotherapy at US oncology clinics for patients clinically similar to those in KEYNOTE-024 (KN024) and KN042 trials, namely, with stage IV NSCLC and PD-L1 tumor proportion score (TPS) $\geq 50 \%$ with unknown or negative status for sensitizing EGFR mutation or $A L K$ translocation; patients with $<6$ months' follow-up until database cutoff were excluded.

- The Kaplan-Meier (KM) time-to-event method was used to estimate median rwToT and the restricted mean rwToT at 6 and 12 months using observed data, together with extrapolated 18- and 24-month rwToT using the best-fitting parametric function, as well as on-treatment rates at 6,12 and 18 months, stratified by Eastern Cooperative Oncology Group performance status (ECOG PS) of $0-1(n=386)$ and ECOG PS $2(n=138)$.

- Pembrolizumab rwTOT was markedly longer for patients with ECOG PS 0-1 (median of 6.9 months; $95 \% \mathrm{Cl}$ : 5.6-8.3) than for those with ECOG PS 2 (median of 2.3 months; $95 \% \mathrm{Cl}: 1.4-3.5$ ); and on-treatment rates were also substantially greater at each time point (12-month on-treatment rate $36.4 \%$ [95\% Cl: $31.2-41.6$ ] vs $16.2 \%$ [95\% Cl: 9.8-24.0] for the ECOG PS 2 cohort).

- Findings for the ECOG PS 0-1 cohort were similar to those for patients enrolled in $\mathrm{KN} 024$, as well as for patients in KN042 restricted to those with stage IV NSCLC and PD-L1 TPS $\geq 50 \%$.

- For the ECOG PS 0-1 cohort, the median rWTOT of 6.9 months approached the median duration of pembrolizumab treatment recorded in KN024 (7.9 months; $95 \% \mathrm{Cl}, 6.2-11.7)$ and was similar to that in KN042 (6.6 months; $95 \% \mathrm{Cl}: 4.9-8.5)$.

- Similarly, the restricted mean rwToT estimated at 24 months for patients with ECOG PS 0-1 (10.5 months; $95 \%$ Cl: 9.4-11.7; Weibull distribution) resembled the 24-month restricted means for duration of pembrolizumab treatment in the clinical trials of 11.0 months (95\% Cl: 9.5-12.5) and 10.4 months (95\% Cl: 9.3-11.5) in KN024 and KN042, respectively.

- The real-world duration of first-line pembrolizumab monotherapy for the trial-eligible population with ECOG PS $0-1$ in this retrospective cohort study was similar to first-line pembrolizumab monotherapy duration for patients enrolled in the KN024 and KN042 trials, while for patients who would have been excluded from the KEYNOTE trials (with ECOG PS 2), the pembrolizumab rwToT was substantially shorter and on-treatment rates substantially lower. 


\section{References}

Papers of special note have been highlighted as: $\bullet$ of interest; $\bullet \bullet$ of considerable interest

1. Reck M, Rodriguez-Abreu D, Robinson AG et al. Pembrolizumab versus chemotherapy for PD-L1-positive non-small-cell lung cancer. N. Engl. J. Med. 375(19), 1823-1833 (2016).

2. $\quad$ KEYTRUDA ${ }^{\circledR}$ prescribing information (4/2019), Merck Sharp \& Dohme Corp., a subsidiary of Merck \& Co., Inc., Kenilworth, NJ, USA. http://www.merck.com/product/usa/pi_circulars/k/keytruda/keytruda_pi.pdf

3. Herbst RS, Baas P, Kim DW et al. Pembrolizumab versus docetaxel for previously treated, PD-L1-positive, advanced non-small-cell lung cancer (KEYNOTE-010): a randomised controlled trial. Lancet 387(10027), 1540-1550 (2016).

4. Reck M, Rodriguez-Abreu D, Robinson AG et al. Updated analysis of KEYNOTE-024: pembrolizumab versus platinum-based chemotherapy for advanced non-small-cell lung cancer with PD-L1 tumor proportion score of 50\% or greater. J. Clin. Oncol. 37(7), 537-546 (2019).

- Recent update of findings through July 2017 of the KEYNOTE-024 clinical trial, studying pembrolizumab therapy for patients with stage IV non-small-cell lung cancer (NSCLC) with PD-L1 TPS $\geq 50 \%$ and no sensitizing $E G F R$ mutation or $A L K$ translocation, and from which patient-level data are reported in this article.

5. Mok TSK, Wu YL, Kudaba I et al. Pembrolizumab versus chemotherapy for previously untreated, PD-L1-expressing, locally advanced or metastatic non-small-cell lung cancer (KEYNOTE-042): a randomised, open-label, controlled, Phase III trial. Lancet 393(10183), 1819-1830 (2019).

- Recently published results of the KEYNOTE-042 trial, from which patient-level data for those with stage IV NSCLC with PD-L1 TPS $\geq 50 \%$ and no sensitizing $E G F R$ mutation or $A L K$ translocation are reported in this article.

6. Friends of Cancer Research (2018). Establishing a framework to evaluate real-world endpoints. https://www.focr.org/publications/establishing-f ramework-evaluate-real-world-endpoints

7. Gong Y, Kehl KL, Oxnard GR, Khozin S, Mishra-Kalyani PS, Blumenthal GM. Time to treatment discontinuation (TTD) as a pragmatic endpoint in metastatic non-small cell lung cancer (mNSCLC): a pooled analysis of 8 trials. J. Clin. Oncol. 36(15_suppl), 9064-9064 (2018).

8. Blumenthal GM, Gong Y, Kehl K et al. Analysis of time to treatment discontinuation of targeted therapy, immunotherapy, and chemotherapy in clinical trials of patients with non-small cell lung cancer. Ann. Oncol. (2019). doi: 10.1093/annonc/mdz060 (Epub ahead of print).

-• Recently published analysis of randomized NSCLC trials finding that time to treatment discontinuation, analogous to our real-world time on treatment end point, is associated with progression-free survival.

9. Berger ML, Curtis MD, Smith G, Harnett J, Abernethy AP. Opportunities and challenges in leveraging electronic health record data in oncology. Future Oncol. 12(10), 1261-1274 (2016).

10. Khozin S, Blumenthal GM, Pazdur R. Real-world data for clinical evidence generation in oncology. J. Natl Cancer Inst. 109(11), djx187 (2017).

11. Yang CF, Hartwig MG, D’Amico TA, Berry MF. Large clinical databases for the study of lung cancer: making up for the failure of randomized trials. J. Thorac. Cardiovasc. Surg. 151(3), 626-628 (2016).

12. Miller RS, Wong JL. Using oncology real-world evidence for quality improvement and discovery: the case for ASCO's CancerLinQ. Future Oncol. 14(1), 5-8 (2018).

13. Sherman RE, Anderson SA, Dal Pan GJ et al. Real-world evidence - what is it and what can it tell us? N. Engl. J. Med. 375(23), 2293-2297 (2016).

- Review of the reasons for needing real-world evidence, as well as approaches to gathering that evidence and its strengths and limitations.

14. Murthy VH, Krumholz HM, Gross CP. Participation in cancer clinical trials: race-, sex-, and age-based disparities. JAMA 291(22), 2720-2726 (2004).

- Study reporting disparities between racial, sex and age profiles of patients enrolled in US cancer trials as compared with patients with cancer in the general population.

15. Prince RM, Atenafu EG, Krzyzanowska MK. Hospitalizations during systemic therapy for metastatic lung cancer: a systematic review of real world vs clinical trial outcomes. JAMA Oncol. 1(9), 1333-1339 (2015).

16. Roche N, Reddel HK, Agusti A et al. Integrating real-life studies in the global therapeutic research framework. Lancet Respir. Med. 1(10), e29-30 (2013).

17. Merck Sharp \& Dohme Corp. Whitehouse Station, NJ, USA. KEYNOTE-024 trial data on file (data cutoff July 10, 2017), ClinicalTrials.gov identifier: NCT02142738.

18. Merck Sharp \& Dohme Corp. Whitehouse Station, NJ, USA. KEYNOTE-0 42 trial data on file (data cutoff February 26, 2018), ClinicalTrials.gov identifier: NCT02220894.

19. Flatiron Health database. https://flatiron.com/real-world-evidence/ 
20. Khozin S, Abernethy AP, Nussbaum NC et al. Characteristics of real-world metastatic non-small cell lung cancer patients treated with nivolumab and pembrolizumab during the year following approval. Oncologist 23(3), 328-336 (2018).

- Early study of the characteristics of patients prescribed pembrolizumab or nivolumab for NSCLC in real-world settings.

21. Curtis MD, Griffith SD, Tucker M et al. Development and validation of a high-quality composite real-world mortality endpoint. Health Serv. Res. 53(6), 4460-4476 (2018).

22. Social Security Death Master File. https://ladmf.ntis.gov/

23. Royston P, Parmar MK. Restricted mean survival time: an alternative to the hazard ratio for the design and analysis of randomized trials with a time-to-event outcome. BMC Med. Res. Methodol. 13, 152 (2013).

24. Zhao L, Claggett B, Tian L et al. On the restricted mean survival time curve in survival analysis. Biometrics 72(1), 215-221 (2016).

25. Pocock SJ, Clayton TC, Altman DG. Survival plots of time-to-event outcomes in clinical trials: good practice and pitfalls. Lancet 359(9318), 1686-1689 (2002).

26. NICE DSU Technical Support Document 14: undertaking survival analysis for economic evaluations alongside clinical trials extrapolation with patient-level data (2013). http://nicedsu.org.uk/wp-content/uploads/2016/03/NICE-DSU-TSD-Survival-analysis.updated-March-2013.v2.pdf

27. Cuyún Carter G, Barrett AM, Kaye JA, Liepa AM, Winfree KB, John WJ. A comprehensive review of nongenetic prognostic and predictive factors influencing the heterogeneity of outcomes in advanced non-small-cell lung cancer. Cancer Manag. Res. 6, 437-449 (2014).

28. van der Linden N, Bongers ML, Coupe VMH et al. Treatment patterns and differences in survival of non-small cell lung cancer patients between academic and non-academic hospitals in the Netherlands. Clin. Lung Cancer 18(5), e341-e347 (2017).

29. Schwartz LH, Litiere S, de Vries E et al. RECIST 1.1-Update and clarification: From the RECIST committee. Eur. J. Cancer 62, 132-137 (2016).

30. Herbst RS, Garon EB, Kim D-W et al. Long-term survival in patients with advanced NSCLC in the KEYNOTE-010 study overall and in patients who completed 2 years of pembrolizumab. Presented at: European Society for Medical Oncology (ESMO) 2018 Congress. Munich, Germany, 19-23 October 2018.

31. Spigel DR, McLeod M, Hussein MA et al. Randomized results of fixed-duration (1-yr) vs continuous nivolumab in patients (pts) with advanced non-small cell lung cancer (NSCLC). Ann. Oncol. 28(Suppl 5), Abstract 1297 O (2017).

32. Spigel DR, McCleod M, Jotte RM et al. Safety, efficacy, and patient-reported health-related quality of life and symptom burden with nivolumab in patients with advanced non-small cell lung cancer, including patients aged [insert "greater than or equal to" symbol] 70 years or with poor performance status (CheckMate 153). J. Thorac. Oncol. (2019) doi:10.1016/j.jtho.2019.05.010 (Epub ahead of print).

33. INSIGNA: a randomized, Phase III study of firstline immunotherapy alone or in combination with chemotherapy in INduction/maintenance or postprogression in advanced nonsquamous non-small cell lung cancer (NSCLC) with immunobiomarker SIGNature-driven Analysis - First-line pembrolizumab alone or in combination with pemetrexed and carboplatin in induction/maintenance or postprogression in treating patients with stage IV non-squamous non-small cell lung cancer (ClinicalTrials.gov Identifier: NCT03793179). https://clinicaltrials.gov/ct2/show/NCT03793179

34. Khushalani NI. Duration of anti-programmed death-1 therapy in advanced melanoma: how much of a good thing is enough? J. Clin. Oncol. 36(17), 1649-1653 (2018). 
